\title{
Measurement of the Analysing Power in Proton-Proton Elastic Scattering
}

Zara Bagdasarian ${ }^{* \dagger}$

Forschungszentrum Jülich, Germany

University of Cologne, Germany

Tbilisi State University, Georgia

E-mail: z.bagdasariandfz-juelich.de

\begin{abstract}
The analysing power in proton-proton (pp) elastic scattering has been measured at small angles for six beam energies between 0.7 and $2.4 \mathrm{GeV}$ using a polarised proton beam and unpolarised proton internal target at COSY-ANKE. The new experimental ANKE results close an important gap in the database of polarised pp elastic scattering.

The analysing power results at $796 \mathrm{MeV}$ agree very well with the corresponding data, which had been obtained in the various experiments around the world. However the ANKE data at the higher energies lie well above the predictions of the most recent published partial wave analysis solution by SAID group (SP07). An updated analysis from SAID, which uses the ANKE results together with the previously available data, leads to a new solution that describes the measurements much better.

The small-angle range, accessible to ANKE and complementary to the EDDA angular range, seems to significantly influence some of the phases and inelasticities in the low-partial waves. This improves the phenomenological understanding of the nucleon-nucleon (NN) interaction, which is not only important on its own, but also will have an impact on understanding of many other NN reactions.
\end{abstract}

53rd International Winter Meeting on Nuclear Physics,

26-30 January 2015

Bormio, Italy

* Speaker.

${ }^{\dagger}$ for the ANKE collaboration 


\section{Introduction}

Protons and neutrons, also known by common name of nucleons, comprise $99 \%$ of the mass of the visible universe. We know, that the interactions between nucleons and their constituent quarks and gluons, are mainly governed by the strong interaction. However, Quantum Chromodynamics, the theory of strong interaction, which perfectly describes the phenomena at high energies, cannot give us quantitative description of the strong interaction at lower energies. Hence the only feasible approach to describe nucleon interaction at intermediate energies is phenomenological one. That is why, the partial wave decomposition of the proton-proton and proton-neutron scattering data is a very important issue in nuclear physics.

The SAID data base and analysis (Scattering Analysis Interactive Dialin) [1] have proved to be a truly invaluable tool over many years for researchers working in this area. Such an analysis is based on the measurements of various NN scattering observables at different energies over the full angular range. Many accelerators around the world included the NN study into their research program, however even after many years of studies, there are still many gaps in our knowledge.

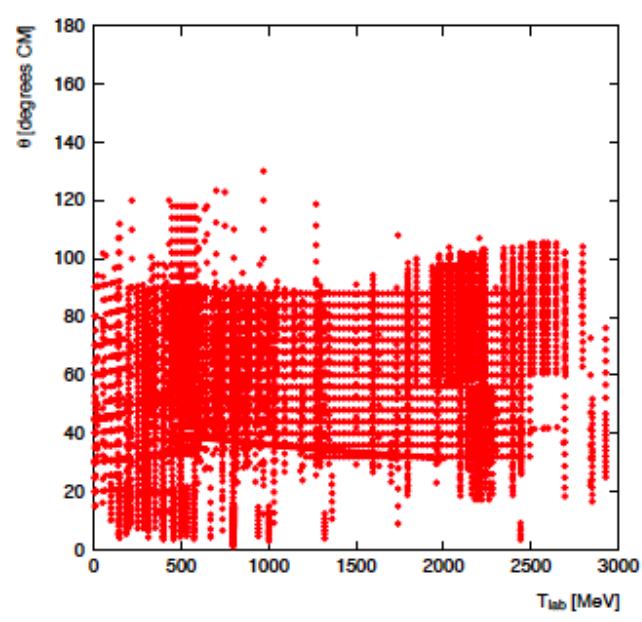

Figure 1: Abundance plot of c.m. scattering angle $\left(\theta_{c m}\right)$ versus beam energy $\left(T_{l a b}\right)$ for experiments on the analysing power for proton proton elastic scattering. Source: nn-online.org

As one can see on the Fig. 1, even in the data base of the most basic reaction of proton-proton elastic scattering, there has been a significant gap at the small angles $\left(\theta_{c m}<30^{\circ}\right)$ above $1 \mathrm{GeV}$. It should be noted, that the wealth of data between $30^{\circ}$ and $90^{\circ}$ comes from the measurements of proton-proton elastic scattering undertaken by the COSY-EDDA collaboration [2]. These measurements have had a major impact on the partial wave analysis of this reaction above $1 \mathrm{GeV}$. In complete contrast to COSY-EDDA, the COSY-ANKE facility was designed for the investigation of the small angle region and is thus well suited to cover this significant gap in the database.

\section{Experiment}

The experiment was carried out using the ANKE magnetic spectrometer [3] positioned inside the storage ring of the COoler SYnchrotron (COSY) [4] of the Forschungszentrum Jülich. Al- 
though the facility sketched in Fig. 2 is equipped with other elements, the only detectors used in this experiment were the forward detector (FD) and the silicon tracking telescopes (STT) [5].

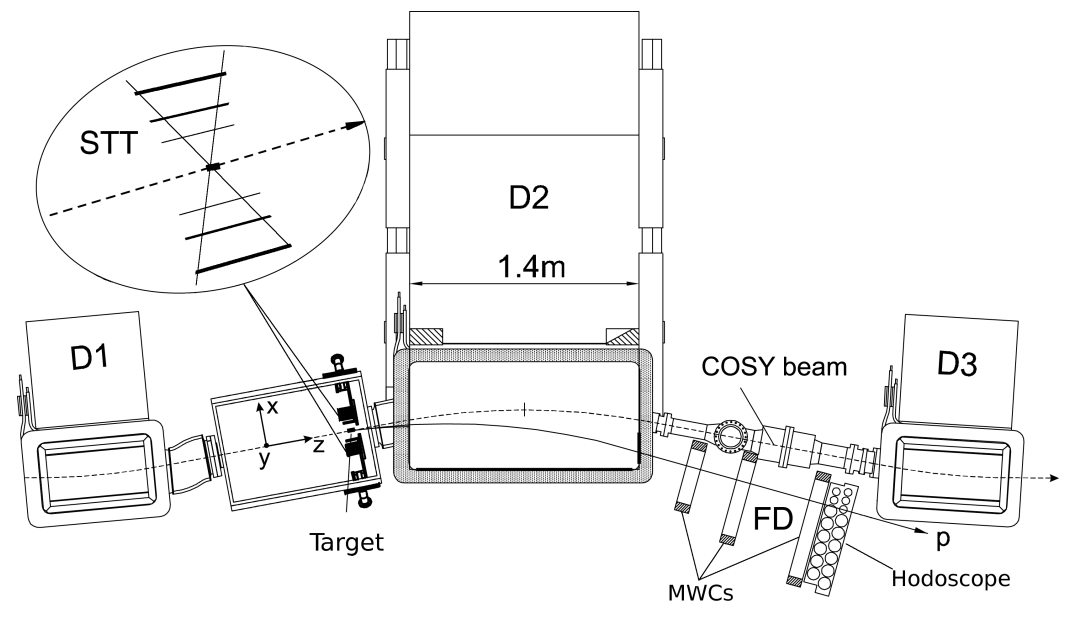

Figure 2: The ANKE spectrometer setup (top view), showing the positions of the hydrogen cluster-jet target, the silicon tracking telescopes (STT), and the forward detector (FD).

The fast protons from elastic $p p$ scattering were measured in the forward detector which, for $p p$ elastic scattering, covered $10^{\circ}-30^{\circ}$ in c.m. polar angles and $\pm 30^{\circ}$ in azimuth. The FD comprises a set of multiwire proportional and drift chambers (MWCs) and a two-plane scintillation hodoscope. The counters were used to measure the energy losses required for particle identification [6]. The two STTs were placed symmetrically inside the vacuum chamber, to the left and right of the beam near the unpolarised hydrogen cluster-jet target [7]. Each telescope consists of three double-sided silicon strip detectors of $70 \mu \mathrm{m}, 300 \mu \mathrm{m}$, and $5 \mathrm{~mm}$ thickness and covers the laboratory polar angles $75^{\circ}<\theta_{\text {lab }}<140^{\circ}$.

The ANKE experiment used a vertically polarised beam incident on an unpolarised target so that the preparation and the measurement of the beam polarisation are critical. The $\mathrm{H}^{-}$ions from the polarised ion source were accelerated to $45 \mathrm{MeV}$ in the cyclotron JULIC before being stripped of their electrons and injected into COSY [8]. Two modes, with spin up $(\uparrow)$ and down $(\downarrow)$, were supplied by the source and the polarisations of the injected beam were optimised using a low energy polarimeter (LEP) in the injection beam line to COSY [9]. The LEP measurements showed that the magnitudes of the polarisations were typically about $93 \%$ and the difference between the values of the two modes was smaller than the statistical uncertainty of $1 \%$.

In a strong-focusing synchrotron, such as COSY, resonances can lead to losses of polarisation of a proton beam during acceleration. In order to compensate for these effects, adiabatic spinflip was used to overcome the imperfection resonances and tune-jumping to deal with the intrinsic ones [10]. The polarisations were measured using the EDDA detector as a polarimeter. This detector, originally equipped with a polarised hydrogen target, had been used to measure the $p p$ analysing power over almost the whole COSY energy range [2]. By studying further the scattering of polarised protons on $\mathrm{C}$ and $\mathrm{CH}_{2}$ targets, it was possible to deduce the quasi-free analysing power of the carbon, where the necessary calibration standard was provided by the EDDA $p \vec{p}$ data [11]. 
The experiment at ANKE was carried out at six energies, $T_{p}=796,1600,1800,1965,2157$, and $2368 \mathrm{MeV}$. Cycles of $180 \mathrm{~s}$ or $300 \mathrm{~s}$ duration were used for each spin mode, with the last $20 \mathrm{~s}$ of each cycle being reserved for the $7 \mu \mathrm{m}$ diameter carbon fibre target to be moved into the beam from below into the EDDA section ${ }^{1}$.

\section{Data Analysis}

The EDDA polarimeter consists of 29 pairs of half-rings placed to the left and right of the beam. It is therefore possible to compare the rates in the left and right half-rings for each range in polar angle $\theta_{\text {lab }}$ while averaging over the azimuthal angle $\phi$ in every half-ring. In order to assure fast polarimetry, the coincidences are recorded by scalers. The asymmetry is determined individually for each pair of half-rings and the weighted average evaluated. The systematic uncertainty of the measurements was estimated to be $3 \%$ at each energy [11]. In order to calculate the analysing power, one has to know the beam polarisation and measure the asymmetry of the elastically scattered protons. The latter was calculated independently using the data taken by FD and STT, while the beam polarisation was determined from the data taken by EDDA-detector at the end of every cycle.

The polarisation weighted averages over time and polar angle are given in Table 1 [12]. The changes in sign reflect the number of spin flips required to pass through the imperfection resonances. The variation of the beam polarisation cycle by cycle was checked with the asymmetry of the counts in STT and found to be around 0.04 (RMS). It should be noted that each of the six beams was prepared independently and, for this reason, the magnitude of the polarisation may not decrease monotonically with the energy.

\begin{tabular}{|c|c|c|c|c|c|c|}
\hline$T_{p}(\mathrm{MeV})$ & 796 & 1600 & 1800 & 1965 & 2157 & 2368 \\
\hline$p$ & 0.554 & 0.504 & -0.508 & -0.429 & -0.501 & 0.435 \\
& \pm 0.008 & \pm 0.003 & \pm 0.011 & \pm 0.008 & \pm 0.010 & \pm 0.015 \\
\hline$N$ & 1.00 & 1.00 & 0.99 & 1.09 & 1.01 & 0.93 \\
\hline
\end{tabular}

Table 1: The values of the mean polarisations $p$ determined with the EDDA polarimeter averaged over all the data at the beam energy $T_{p}$ where the $p p$ analysing power was measured in ANKE. The changes in sign in $p$ are due to the spin flips induced when passing through the imperfection resonances. Though the shown statistical errors are small, there are $3 \%$ systematic uncertainties [11]. The normalisation factors $N$ are those obtained in a partial wave fit [1] to the current STT data, as discussed in the text.

In the ANKE experiment a proton is detected in either the STT or FD and elastic $p p$ scattering events are identified through the evaluation of the missing mass in the reaction. As can be seen from typical examples of both cases shown in Fig. 3 at a beam energy of $1.6 \mathrm{GeV}$, there is very little ambiguity in the isolation of the proton peak. The greater suppression of events associated with pion production in the STT is due to the minimum longitudinal momentum of the recoil proton and the restricted angular acceptance of this detector.

\footnotetext{
${ }^{1}$ The EDDA target effectively consumes all the beam so that it could not be used before an ANKE measurement in a cycle.
} 


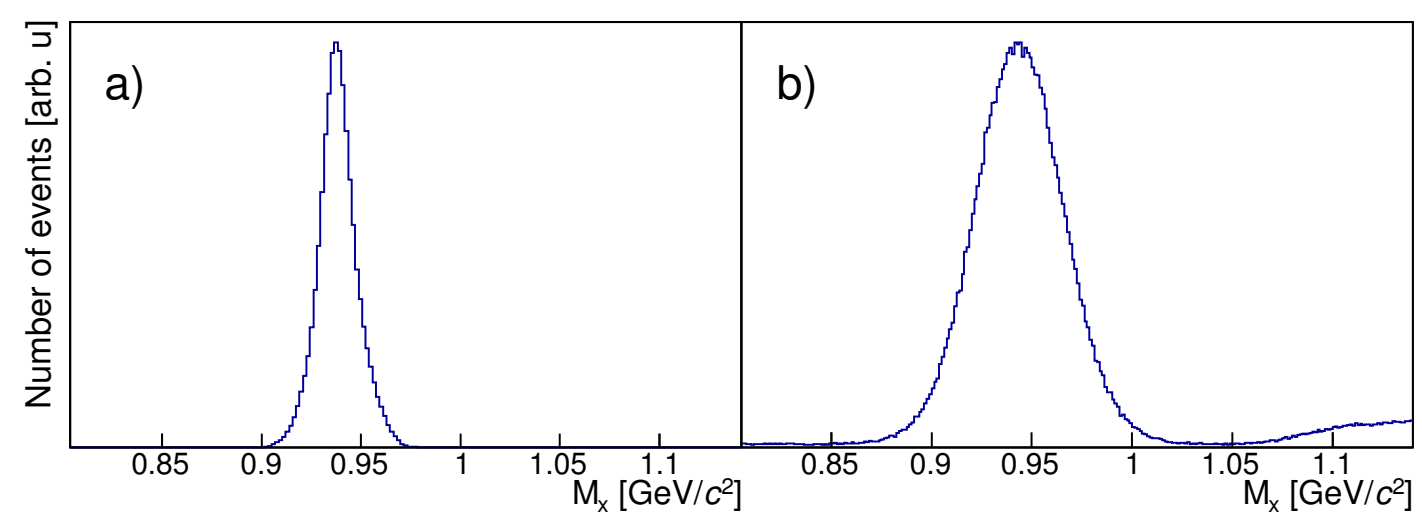

Figure 3: Missing-mass $M_{X}(p p \rightarrow p X)$ spectra obtained for a beam energy of $1.6 \mathrm{GeV}$ showing the clear proton peak when detecting one proton in (a) the STT and (b) the FD.

The left/right symmetry of the STT system allows one to use the so-called cross-ratio method [14]. It eliminates first-order systematic errors that arise from misalignments between the two STT and it is for this reason that the beam polarisation was reversed in each successive cycle. Let $L_{\uparrow}\left(L_{\downarrow}\right)$ be the numbers of counts in the left telescope with spin up (down) and $R_{\uparrow}\left(R_{\downarrow}\right)$ the analogous quantities for the right telescope. In terms of the geometric means, $L=\sqrt{L_{\uparrow} R_{\downarrow}}$ and $R=\sqrt{R_{\uparrow} L_{\downarrow}}$, the scattering asymmetry is related to the analysing power $A_{y}(\theta)$ for each value of the scattering angle $\theta$ through

$$
\varepsilon(\theta)=\frac{L(\theta)-R(\theta)}{L(\theta)+R(\theta)}=A_{y}(\theta) p\langle\cos \phi\rangle
$$

where $p\langle\cos \phi\rangle$ is the effective beam polarisation, taking into account the acceptance of the STT in the azimuthal angle $\phi$. In our geometry $\langle\cos \phi\rangle \approx 0.966$.

Other systematic errors, such as those arising from differences in the magnitudes of the up and down polarisations, also cancel in first order. The overall systematic uncertainty in $A_{y}$ arising from asymmetry measurement with STT does not exceed $0.3 \%$. Another factor that could affect the asymmetry measured with such a two-arm detector is any instability in the ratio of the efficiencies of the left and right telescopes. The instability correction, which was studied at all energies, does not exceed the $|c|=1.3 \%$ that was found at $1.8 \mathrm{GeV}$. The relevant corrections of the analysing power $c(\theta) A_{y}(\theta)$ were added for each angular bin [13].

FD is a single-sided detector, so the measured asymmetry is more sensitive to the distortion factors like the beam polarisation modules inequality and geometry misalignments. The number of events for each orientation of the polarisation was weighted with the relative luminosity factors, which were fixed by comparing the rates of charged particle production in angular regions where the beam polarisation could play no part [15]. Consistent values for the relative luminosities were achieved when varying these cuts and it is estimated that the systematic uncertainty of $A_{y}$ due to the relative luminosity normalisation never exceeds $0.3 \%$. This approach could be checked by comparing the FD and STT results in the angular overlap regions.

The efficiency for registering events in the forward detector induced by spin-up or spin-down protons was studied by using events where both the fast and recoil protons were measured in the 
FD and STT, respectively. The differences of the efficiencies of less than $10^{-3}$ could be neglected compared to the statistical uncertainties. Potentially more serious for the FD analysis is the assumption that the magnitudes of the two polarisation modes were identical, viz. $\left|p_{\uparrow}\right|=\left|p_{\downarrow}\right|$. Whereas deviations from the mean are very small at injection, and are known to be less than 5\% after acceleration, these could induce fractional errors in $A_{y}$ of up to $2.5 \%$. It should, however, be remarked that in the overlap regions of the STT and FD data any disagreements between the determinations of the asymmetries in the two systems are on the $1 \%$ level and this puts a much tighter constraint on possible $\left|p_{\uparrow}\right|,\left|p_{\downarrow}\right|$ differences.

In cases where one of the protons from an elastic scattering event is detected in the FD and the other in the STT it is possible to compare directly the scattering angle measured in the two systems. In general $\theta_{c m}(\mathrm{STT})>\theta_{c m}(\mathrm{FD})$, with the difference being typically $\approx 0.3^{\circ}$. It is not possible to judge which detector is responsible for this difference which is, however, small compared with the bin widths of $1.0^{\circ}(\mathrm{FD})$ and $1.2^{\circ}$ (STT).

The dominant systematic error is that arising from the determination of the beam polarisation in the EDDA polarimeter, which was estimated to be 3\% [11]. For the FD data there is, in addition, a possible contribution associated with the assumption of equal up and down polarisations so that in this case we would cautiously assume a 5\% systematic uncertainty. To these figures must be added the statistical uncertainty in the determinations of the beam polarisations at the six energies shown in Table 1.

\section{Results}

The results of all the ANKE measurements of $A_{y}$ for $p p$ elastic scattering are shown for the six energies in Fig. 4. For the five of these energies the measurements have been performed for the first time in the angular range from $4^{\circ}$ to $28^{\circ}$. The agreement between the STT and FD data, which involved completely independent measurements of the final state, is remarkably good. The individual deviations generally lie within the statistical error bars and the average over the angular overlap regions is $A_{y}(\mathrm{FD}) / A_{y}(\mathrm{STT})=1.00 \pm 0.01$. Though the overall uncertainties are slightly larger for the FD data, these results are important because they extend the coverage to slightly larger scattering angles.

At beam energies close to $796 \mathrm{MeV}$ there are many measurements of the $p p$ analysing power and, in general, they are in a very good mutual agreement, as they are with the new ANKE data. This reinforces the confidence in the use of the EDDA polarimeter. At $1.6 \mathrm{GeV}$ and above there are far fewer experimental measurements and, for clarity, we only show the EDDA data at neighbouring energies though, at the highest energy, the statistical fluctuations are significant [2]. The SAID SP07 solution [1], shown by the solid black line in Fig. 4, describes the bulk of the $\approx 796 \mathrm{MeV}$ data very well indeed. However, at higher energies the ANKE data deviate significantly from the predictions of the SP07 solution. Moreover, the shapes of the ANKE data seem very different from these predictions, rising much more steeply at small angles. Therefore, these discrepancies cannot be due to a simple miscalibration of the EDDA polarimeter, for example, which would change the overall magnitude of $A_{y}(\theta)$ but not its angular dependence.

The ANKE analysing power data have been added to the World data set and searches made for an updated partial wave solution [1]. To allow for possible systematic effects, the SAID fitting 

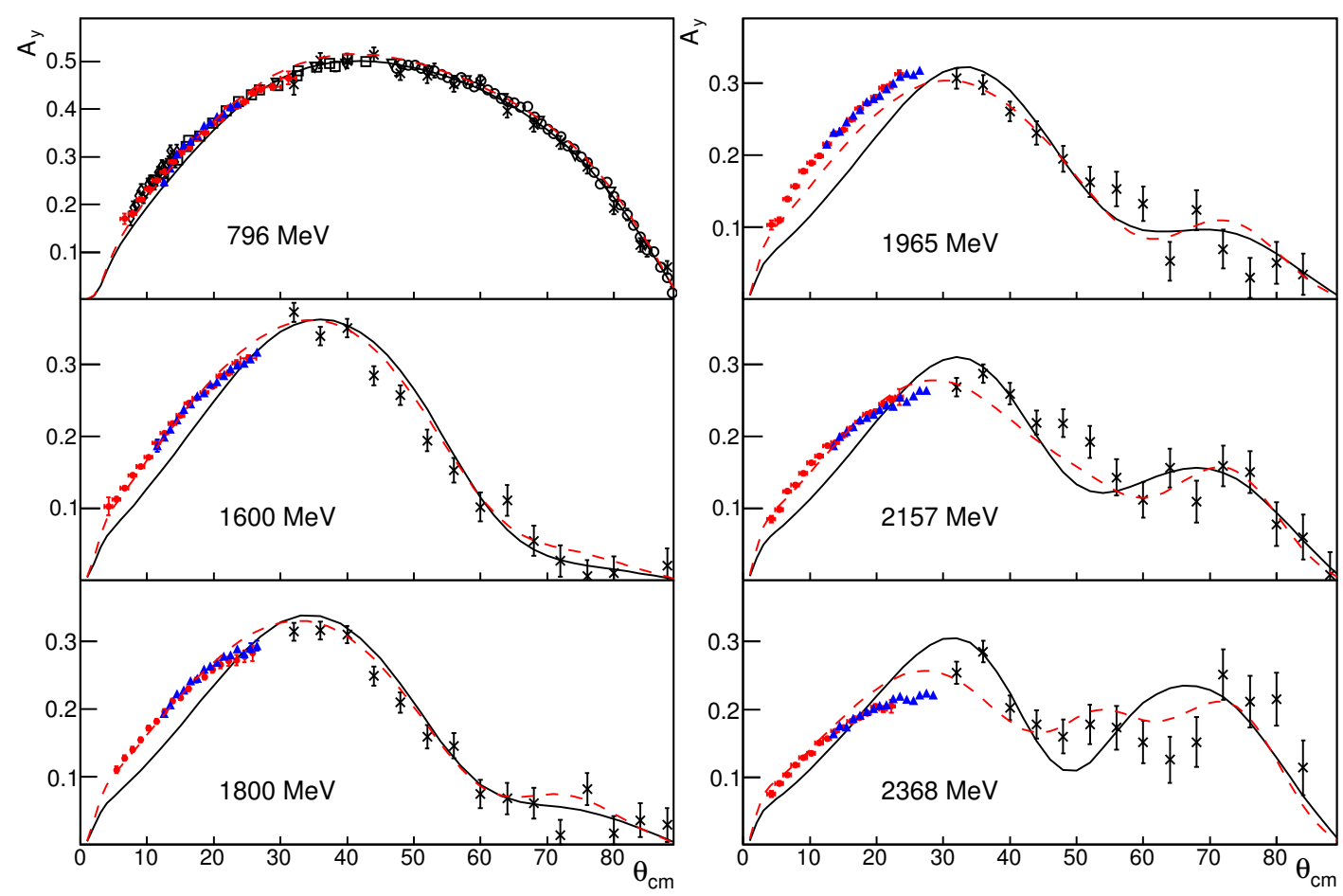

Figure 4: Comparison of the ANKE measurements of the proton analysing power in $p p$ elastic scattering using the STT (red filled circles) and FD (blue filled triangles) systems with the curves corresponding to the SAID SP07 (solid black line) and the revised fit (dashed red) solutions [1]. Only statistical errors are shown so that the systematic uncertainties arising, for example, from the calibration of the EDDA polarimeter have not been included. Also shown are selected results from EDDA (black crosses) [2] at the energies different by no more than $7 \mathrm{MeV}$ and, at $796 \mathrm{MeV}$, LAMPF [16, 17, 18], and SATURNE [19] (black open symbols). It should be noted that the EDDA data were taken over a continuous ramp [2] and, if continuity in energy were imposed, many of their statistical fluctuations would be diminished.

procedure introduces a scale factor $N$ into any data set and determines its value, as well as the $p p$ phases and inelasticities, by minimising an overall $\chi^{2}$ for the complete data set. The new fits, which lead to the red dashed curves in Fig. 4, correspond to relatively modest changes to the parameters of the lower partial waves. The values of the normalisation factors $N$ reported in Table 1 have an average of $\langle N\rangle=1.00 \pm 0.02$ for the STT data. These factors, which would effectively multiply the beam polarisations, have not been applied in Fig. 4. The deviations of the individual values of $N$ from unity might seem to be greater at the higher energies. They are somewhat larger than what one would expect on the basis of the quoted uncertainties in the EDDA polarimeter, being around $5 \%$ rather than the $3 \%$ estimate [11]. It should be stressed that the introduction of the scale factor $N$ does not change the shape of a distribution and, even in cases where a value close to one is found, this does not mean that the fit reproduces perfectly the data. A clear example of this is to be found in the larger angle data at $1.6 \mathrm{GeV}$ shown in Fig. 4.

In summary, in the small angle range accessible to ANKE, the new data are consistent with older measurements around $796 \mathrm{MeV}$ and also with the SP07 SAID predictions at this energy [1]. At higher energies the ANKE results lie significantly above the SP07 solution near the forward 
direction and also display a different angular dependence. By adjusting some of the phases and inelasticities in the low partial waves of this solution it has been possible to obtain a much better description of the ANKE $A_{y}$ data with reasonable values of $\chi^{2} / N D F$. The new fits correspond to relatively modest changes to the parameters for several of the lower waves, with the biggest change being in ${ }^{3} F_{2}$.

\section{Acknowledgements}

This work has been partially financed by the Shota Rustaveli National Science Foundation of Georgia.

\section{References}

[1] R.A. Arndt, I.I. Strakovsky, R.L. Workman,Phys. Rev. C 62 (2000) 034005;

R.A. Arndt, W.J. Briscoe, I.I. Strakovsky, R.L. Workman, Phys. Rev. C 76 (2007) 025209;

http://gwdac.phys.gwu.edu.

[2] M. Altmeier et al., Phys. Rev. Lett. 85 (2000) 1819 ; M. Altmeier et al., Eur. Phys. J. A 23 (2005) 351.

[3] S. Barsov et al., Nucl. Instrum. Methods A 462 (2001) 364 .

[4] R. Maier et al., Nucl. Instrum. Methods A 390 (1997) 1 .

[5] R. Schleichert et al., IEEE Trans. Nucl. Sci. 50 (2003) 301.

[6] S. Dymov et al., Part. Nucl. Lett. 2(119) (2004) 40.

[7] A. Khoukaz et al., Eur. Phys. J. D 5 (1999) 275.

[8] P.D. Eversheim et al., AIP Conf. Proc. 293 (1993) 92.

[9] D. Chiladze et al., Phys. Rev. ST Accel. Beams 9 (2006) 050101.

[10] A. Lehrach et al., AIP Conf. Proc. 675 (2003) 153.

[11] E. Weise, PhD thesis, University of Bonn, 2000.

[12] Z. Bagdasarian et. al., Phys. Let. B 739 (2014) 152.

[13] Z. Bagdasarian, S. Dymov, and G. Macharashvili, ANKE internal reports available from collaborations.fz-juelich.de/ikp/anke/internal.shtml.

[14] G.G. Ohlsen, P.W. Keaton Jr., Nucl. Instrum. Methods 109(1973) 41.

[15] S. Yaschenko et al., Phys. Rev. Lett. 94 (2005) 072304.

[16] M.W. McNaughton et al., Phys. Rev. C 23 (1981) 1128.

[17] F. Irom, G.J. Igo, J.B. McClelland, C.A. Whitten, Phys. Rev. C 25 (1982) 373.

[18] P.R. Bevington et al., Phys. Rev. Lett. 41 (1978) 384.

[19] C.E. Allgower et al., Nucl. Phys. A 637 (1998) 231. 